\title{
Microphase separation in linear multiblock copolymers under poor solvent conditions
}

\author{
Panagiotis E. Theodorakis, ${ }^{* a}$ and Nikolaos G. Fytas ${ }^{b}$
}

Received Xth $X X X X X X X X X X 20 X X$, Accepted Xth $X X X X X X X X X 20 X X$

First published on the web $X t h X X X X X X X X X X 200 X$

DOI: 10.1039/b000000x

Molecular dynamics simulations are used to study the phase behavior of linear multiblock copolymers with two types of "monomers, A and B, where the length of the polymer blocks $N_{A}$ and $N_{B}\left(N_{A}=N_{B}=N\right)$, the number of the blocks $n_{A}$ and $n_{B}\left(n_{A}=n_{B}=n\right)$, and the solvent quality varies. The fraction $f$ of A-type monomers is kept constant and equal to 0.5 . Whereas 'at high enough temperatures these macromolecules form coil structures, where each block A or B forms rather individual clusters, at low enough temperatures A and B monomers from different blocks can join together forming clusters of A or B monomers. The dependence of the formation of these clusters on the varied parameters is discussed in detail, providing a full understanding of the phase behavior of linear multiblock copolymers, at least for this symmetrical case.

\section{Introduction}

The phase separation behavior of block copolymers has been the subject of several theoretical and experimental studies. In the case of infinitely dilute solutions, it is sufficient to evaluate the behavior of isolated chains. Very interesting is the case of multiblock copolymers with blocks composed of either A- or B-type monomers for which very successful methods of their synthesis exist. $\frac{31,32}{}$ Apart from the experimental characterization, numerical investigations of model chains are the most direct approach to understanding the behavior of these 'systems. ${ }^{33-36}$ Interestingly, for the case of multiblock copolymers there is also close relation to the various toy-models '(i.e., the HP model ${ }^{37-42}$ ), which try to mimic the behavior of biomacromolecules on the way to understanding complicated biological processes, i.e., protein folding, helical structures ${ }^{43}$, etc.

Multiblock copolymers of two chemically different type of blocks (A and B) are expected to adopt at high temperatures 'coil structures, where chain conformations are essentially governed by repulsive interactions between the different blocks. The result is an expansion of the chain dimensions, not only with respect to the unperturbed state, but also with respect to a homopolymer of the same length under the same thermodynamic conditions. ${ }^{44}$ Also, one expects that the spherical symmetry of these macromolecules should break, so that the chain forms a slightly elongated object, but such phenomena will not be discussed in the present manuscript. Here, we focus on the microphase separation between the blocks of different

a Institut für Physik, Johannes Gutenberg Universität, Mainz, Germany. Email: theodora@uni-mainz.de

${ }^{b}$ Department of Materials Science, Patras 26504, Patras, Greece. type in a multiblock copolymer chain with two types of blocks (A and B) and the dependence on its structural parameters in terms of their cluster analysis. In this direction, the most interesting properties of such macromolecules are obtained when the solvent quality varies. Under poor solvent conditions (temperatures lower than the Theta temperature of the chain, which in our case will be the same for two different types of blocks due to the symmetry of our model) the chain collapses forming globular structures, where microphase separation between different blocks takes place. The different blocks A and B come together and form clusters of monomers of type A or B, which are microphase separated. In this study, we analyze these phenomena by studying the formation of these clusters and leave the discussion of their size, in conjunction with the size of the individual blocks, for a later communication. To this end, we have performed large-scale molecular dynamics (MD) simulations of an off-lattice model of a linear multiblock copolymer varying the solvent quality to provide a first approach to the understanding of phase separation of such macromolecules.

The outline of this paper is as follows. Sec. 2 describes our model and sketches the analysis needed to characterize the size and overall shape (and other properties) of the clusters considering average properties. Then, Sec. 3 presents our numerical results and Sec.4 summarizes our conclusions.

\section{Model and simulation methods}

In this study we consider the most symmetric case of multiblock copolymers, where the fraction of monomers of type A is $f=0.5$. Then the length of blocks $\mathrm{A}, N_{A}$, and blocks $\mathrm{B}, N_{B}$, is equal as well, and the number of blocks $n_{A}$ with monomers of type $\mathrm{A}$ and $n_{B}$ with monomers of type $\mathrm{B}$ is the 
same. In this study we denote the total number of blocks as $n\left(n=n_{A}+n_{B}\right)$ and the length of the blocks with the capital letter $N\left(N=N_{A}=N_{B}\right)$. Figure 1 shows schematically the definition of the above parameters. Then, the total length of the multiblock chain is $n N$. Clearly, this choice facilitates the study of this problem, being the most symmetric case. Our chains are modelled by the standard bead-spring model,,$\underline{45}-\underline{47}$ where all beads interact with a truncated and shifted LennardJones (LJ) potential

$$
U_{L J}(r)=\left\{\begin{array}{lr}
4 \varepsilon_{L J}\left[\left(\sigma_{L J} / r\right)^{12}-\left(\sigma_{L J} / r\right)^{6}\right]+C, & r \leq r_{c} \\
0 & , r>r_{c}
\end{array}\right.
$$

where $r_{c}=2.5 \sigma_{L J}$ is the cut-off of the potential, and the constant $C$ is defined such that $U_{L J}\left(r=r_{c}\right)$ is continuous at this cut-off. Henceforth, units are chosen such that $\varepsilon_{L J}=1$, $\sigma_{L J}=1, k_{B}=1$, and $m=1$ (mass of the beads) for simplicity. When we consider two types $(\mathrm{A}, \mathrm{B})$ of blocks, we still use $\sigma_{L J}^{A A}=\sigma_{L J}^{A B}=\sigma_{L J}^{B B}=1$ and $\varepsilon_{L J}^{A A}=\varepsilon_{L J}^{B B}=1$ but $\varepsilon_{L J}^{A B}=1 / 2$ to create an unmixing tendency $\left(\Delta \varepsilon=\varepsilon_{A B}-1 / 2\left(\varepsilon_{A A}+\varepsilon_{B B}\right)\right)$. We know that in the case of a binary system with monomers at density $\rho=1$ (e.g., a LJ mixture which is a standard system for the study of phase separation), microphase separation occurs below a critical temperature $T_{c}$ close to $T=1.5 . \underline{\underline{48}}$ For the multiblock copolymers the average densities are much smaller, but since the critical temperature scales proportional to the chain length $\left(T_{c} \propto \chi_{c r i t}^{-1}\right)$, we do expect to be able to detect microphase separation with our model. For this we also exploit previous experience with this model where microphase separation was studied in the case of bottlebrush polymers with two types of side chains. $\underline{51}$

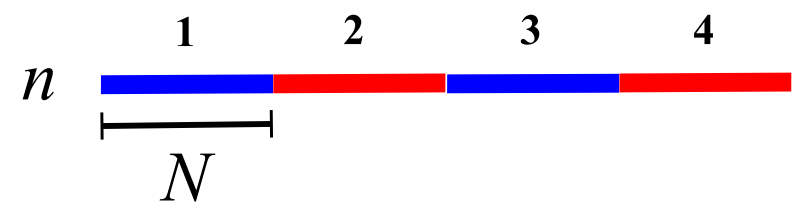

Fig. 1 Definition of structural parameters describing our multiblock copolymer chains. $n$ is the number of different blocks A and B (in this case $n=4$ ) and $N$ is the length of each block. All the blocks, irrespective of whether they are of type A or B, have the same length $N$. Then, the total length of the chain is $n N$.

The connectivity of the beads along the chain is maintained by the finitely extensible nonlinear elastic (FENE) potential

$$
U_{F E N E}(r)=-\frac{1}{2} k r_{0}^{2} \ln \left[1-\left(r / r_{0}\right)^{2}\right], 0<r \leq r_{0},
$$

where the standard choice of parameters $\left(r_{0}=1.5\right.$ and $\left.k=30\right)$ was adopted, and $U_{F E N E}\left(r>r_{0}\right)=\infty$.

For the model defined by Eqs. (1) and (2) the Theta temperature is known only rather roughly, ${ }^{49}$ namely $\Theta \approx 3.0$. Being

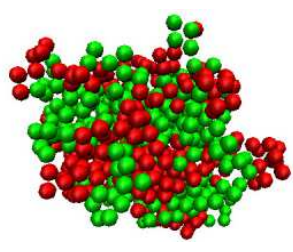

(a)

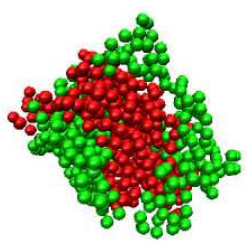

(b)

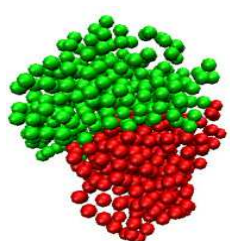

(c)
Fig. 2 Snapshots of three different multiblock copolymers of the same total length $n N=600$ at a low temperature $T=1.5$ ((a): $N=6$, (b): $N=15$, and (c): $N=60$ ). In case (c) we have the formation of two clusters of different blocks which are always phase separated.

interested in $T \leq \Theta$, we have attempted to study the temperature range $1.5 \leq T \leq 3.0$. However, as it is well-known, equilibration of collapsed chains is a rather difficult task and our simulation procedure will be discussed below. The temperature is controlled by the Langevin thermostat, as done in previous work. $\stackrel{45-47.49}{ }$ The equation of motion for the coordinates $\left\{\vec{r}_{i}(t)\right\}$ of the beads

$$
m \frac{d^{2} \vec{r}_{i}}{d t^{2}}=-\nabla U_{i}-m \gamma \frac{d \vec{r}_{i}}{d t}+\vec{\Gamma}_{i}(t)
$$

is numerically integrated using the GROMACS package. $\frac{50}{} \mathrm{In}$ Eq. (3) $t$ denotes the time, $U_{i}$ is the total potential the $i$-th bead experiences, $\gamma$ is the friction coefficient, and $\vec{\Gamma}_{i}(t)$ the random force. $\gamma$ and $\vec{\Gamma}_{i}(t)$ are related by the fluctuation-dissipation relation as follows

$$
\left\langle\vec{\Gamma}_{i}(t) \cdot \vec{\Gamma}_{j}\left(t^{\prime}\right)\right\rangle=6 k_{B} T \gamma \delta_{i j} \delta\left(t-t^{\prime}\right) .
$$

As in previous work, $, 45-47,49,51-53$ the friction coefficient was chosen $\gamma=0.5$. For the integration of Eq. (3) the leap frog algorithm ${ }^{54}$ was used with a time step of $\Delta t=0.006 \tau$, where the natural time unit is defined as $\tau=\left(m \sigma_{L J}^{2} / \varepsilon_{L J}\right)^{1 / 2}=1$.

As we mentioned earlier, equilibration of collapsed chains via MD methods is difficult, and thus we briefly describe here our equilibration procedure. First the system was equilibrated at $T=3.0$ for a time range of $30 \times 10^{6} \tau$. To gather statistics, a sufficient number of statistically independent configurations (typically greater than 500) at this temperature was used for initial configurations of slow cooling runs, where the temperature $T$ was lowered in steps of 0.1 , and running the system at each $T$ for a time which exceeds the relaxation time of our chains. The final configuration of each (higher) $T$ was used as starting configuration for the next (lower) $T$. In this way, we are able to generate statistically independent configurations of clusters. One can see already from snapshot pictures of Fig. 2 the different structures that occur at a low temperature $T=1.5$ for different multiblock chains, where $n N$ is constant, but $N$ varies. Indeed, rather dense clusters containing several blocks 
occur, and the conformation depends on the parameters $N, n$, and $T$ (remember that $\chi N \propto \Delta \varepsilon / T$ ). We also note that as the length of the chains increases, equilibration of the chains becomes extremely difficult preventing the study of very long chains. With our choice of parameters, we expect to observe the most interesting effects in the range of simulated chain lengths used in this study. In particular, we study in detail the chain length $n N=600$ and then we discuss the dependence on $n$ and $N$ separately, with $N$ of course being the main parameter controlling the incompatibility between $\mathrm{A}$ and $\mathrm{B}$, for the chosen set of (potential) parameters.

When the chains collapse at the lower temperatures to form microphase separated cluster structures, there is no reason a priori for the system to decide which blocks will belong to a specific cluster. We actually observe fluctuations where a block of type A or B that was part of a cluster of similar monomers escapes from the cluster to become part of another neighboring cluster with blocks of the same type of monomers. In fact, many such fluctuations would amount to influencing the characteristic properties (number $N_{c l}$ of blocks a cluster contains, and its linear dimensions) of each cluster.

For the analysis of the clusters one needs to identify for each configuration that is analyzed, which blocks belong to which cluster. We have used the standard Stillinger ${ }^{55}$ neighborhood criterion for monomers: if two monomers are less than a distance $r_{n}$ apart, they belong to the same cluster. We followed the standard choice $r_{n}=1.5 \sigma_{L J}$ and checked that qualitatively very similar results were obtained if one chooses $r_{n}$ a bit smaller than this choice (larger values of $r_{n}$ are physically hardly significant, since then the particles are too weakly bound, due to the rapid fall-off of the LJ potential). The same distance $r_{n}$ is considered also for the analysis of the number of contacts. Therefore, a pair of monomers being an absolute distance less than $r_{n}=1.5$ apart define a "contact". Then, the numbers presented in this manuscript denote the average number of neighbors per monomer.

In the case of properties as it is, for example, the number of contacts, one just considers the thermal average. However, to define properties related to the fluctuating number of clusters one should also take into account averages of quantities $A$ that depend on these fluctuations, i.e.,

$$
\bar{A}=\sum_{N_{c l}} P\left(N_{c l}\right) A\left(N_{c l}\right)
$$

As an example, Fig. 3 shows data of the distribution in the number of clusters $N_{c l}$ for several choices of $n, N$. The extreme choices would be that every block forms a separate cluster $\left(P\left(N_{c l}\right)=1\right.$ for $N_{c l} / n=1$, where $\mathrm{A}$ and $\mathrm{B}$ are always phase separated), which is seen for none of the cases presented in Fig. 3 (the case $n=10$ at temperature $T=3$ shows that this case is possible with a probability $\approx 0.6$ ), and that all blocks of A-type monomers form a cluster with the blocks of B-type

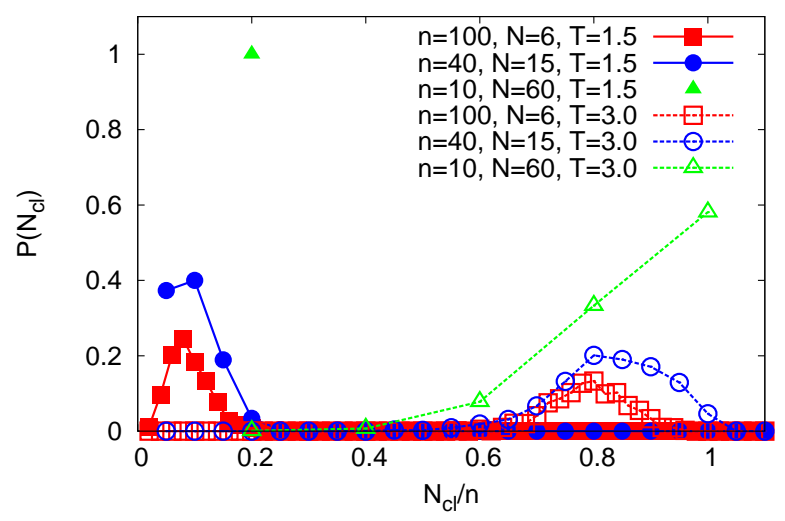

Fig. 3 Probability distribution $P\left(N_{c l}\right)$ of the number of clusters plotted versus $N_{c l} / n$ for combinations of $n$ and $N$ that correspond to the snapshots of Fig. 2 Results are shown for two temperatures, $T=1.5$ and $T=3.0$. Lines are guides for the eye.

forming another cluster $\left(P\left(N_{c l}\right)=1\right.$ for $\left.N_{c l} / n=2 / n\right)$. The latter case is taking place for $n=10$ at $T=1.5$, where we see a single point showing that all blocks of type A are always belonging to the same cluster and all blocks of type B to another cluster. The other cases presented in Fig. 3 are intermediate ones, where we have a symmetric variation in the number of clusters around an average value.

\section{Results and discussion}

The incompatibility between A and B blocks can increase the overall chain dimensions of the chains compared to the homopolymer chain of the same length under the same thermodynamic conditions. A and B monomers prefer to be apart, increasing in this way the overall size of the macromolecule. This can be readily checked if we measure the overall density profile of the multiblock copolymers relative to the center of mass for various cases of $n(n \propto 1 / N$ for $n N=$ const $)$. In Fig. 4 we present this dependence on $n$ for $n N=600=$ const at a low temperature, i.e., $T=1.5$. The total chain length $(n N=600)$ is high enough to discuss the different scenarios of microphase separation in these macromolecules as the length of the blocks varies up to relatively large values of $N$. Indeed, we can see from Fig. 2 that already for $N=60$ the formation of single clusters takes place. Then we can focus on the variation with $n$ and $N$, with the latter parameter being of course mostly important.

The first quantity we have computed is the overall monomer density, mathematically expressed by the following formula

$$
\rho(|\vec{r}|)=\left\langle\sum_{i=1}^{n N} \delta\left(\vec{r}-\vec{r}_{c}-\vec{r}_{i}\right)\right\rangle,
$$




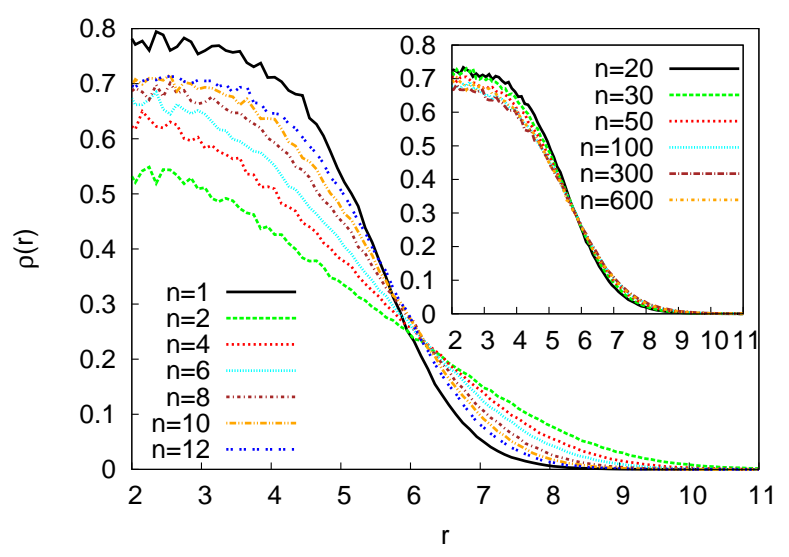

Fig. 4 Density profile $\rho(r)$ plotted versus the radial distance $r$ from the center of mass of the chain at temperature $T=1.5$. Note the pronounced differences occurring for small $n$, whereas the differences for $n>12$ are minor.

where $\delta(\vec{x})$ is the Dirac delta function, $\vec{r}_{c}$ the position of the center of mass of the whole chain, and $\vec{r}_{i}$ the positions of all monomers, irrespective of their type (A or B). The angle brackets denote an average over all conformations as usual. Figure 4 shows the results for the overall monomer density, where $n$ is varied, but $n N$ is kept constant and equal to 600 . These results refer to a temperature $T=1.5$. We can clearly see that the homopolymer chain $(n=1)$ of length $(n N=600)$ obtains always the most compact conformations, if we compare it with the respective cases of multiblock chains. The case of $n=2$ exhibits the greatest difference from the homopolymer case, with the former showing bigger dimensions of the chain, due to the formation of a large A-B interface. As $n$ increases up to $n=12$ the multiblock chains gradually exhibit more compact structures, but they are always more swollen than the homopolymer case. For the range of $n$ between 20 and 600 shown as an inset in Fig. 4 we see that the differences in the density profiles for different multiblock chains are rather small. However, for this range of values $(n=20-600)$ we observe a monotonic behavior, where the chains obtain less compact structures with increasing $n$. This is clearly due to the increase of the unfavorable interactions between $\mathrm{A}$ and B monomers. Actually, we note here that the increase of $n$ should result in breaking of the spherical symmetry, but such effects will not be discussed in this manuscript. Here, we are only concerned with the phase separation of multiblock copolymers under poor solvent conditions. When the density profiles of these systems at significantly higher temperatures (and closer to the Theta temperature, but still under poor solvent conditions) are plotted, somewhat smaller differences occur and the behavior observed at high $N$ is slightly different. These effects will be explained below as we will discuss in

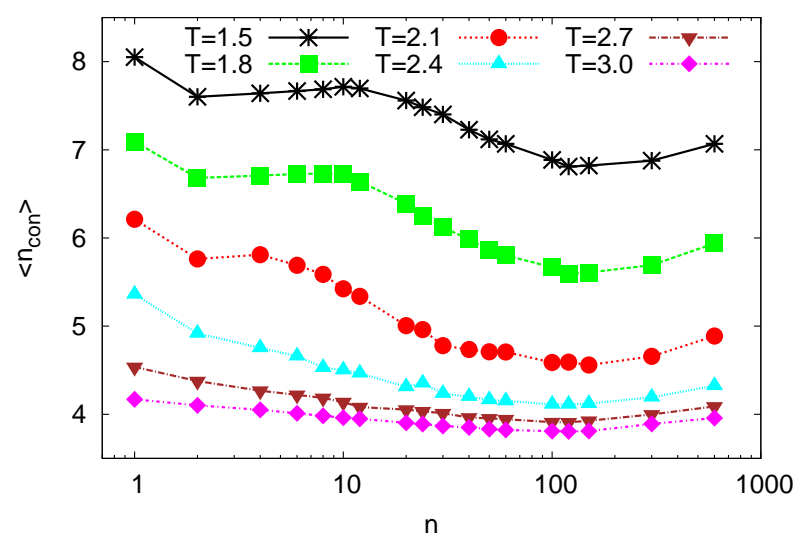

Fig. 5 Average number of contacts $\left\langle n_{\text {con }}\right\rangle$ per monomer plotted versus the number of blocks $n$ for different temperatures distinguished with different symbols and line formats. Note the logarithmic scale in the $x$-axis and the different regions that appear reflecting the different behavior of multiblock copolymers with different $n$.

detail the effect of the temperature.

As $n$ increases, it is natural that the number of contacts between $\mathrm{A}$ and $\mathrm{B}$ monomers increases and then the number of contacts A-A and B-B will decrease. Therefore, we are interested in the overall number of contacts per monomer irrespective of whether a monomer is of type A or B. These results are presented in Fig.5 for different temperatures. We observe that $n_{\text {con }}$ is higher in the case of the homopolymer chain. This confirms our understanding for the homopolymer case, already seen in the calculation of the overall profile. The homopolymer chain is always the most compact globule at this low temperature $T=1.5$ and of course no multiblock chain can obtain such a compact structure that the overall contacts are mostly favored. Moreover, the results of Fig. 5 show that this is true for all temperatures up to $T=3.0$. Therefore, the effect of incompatibility between A and B is rather weakly present at higher temperatures, as expected. These effects would become less noticeable as the temperature increases and becomes considerably higher than the Theta temperature they should be unnoticeable. For temperature $T=1.5$ and small $n$ the curve has a plateau from $n=2$ to $n=10$. This result is surprising, since it shows that the globule at this low temperature has bigger dimensions, but the overall number of contacts stays almost constant as the effect of the solvent incompatibility sets in strongly. Although there are obvious differences in the density profiles of Fig. 4, the multiblock chain for small $n$ rearrange in such a way that the number of contacts stays almost constant. For values higher than $n=12$ the number of contacts decreases and in the case of very high $n(n>100)$ we see a small increase. In the latter case, we notice that the num- 


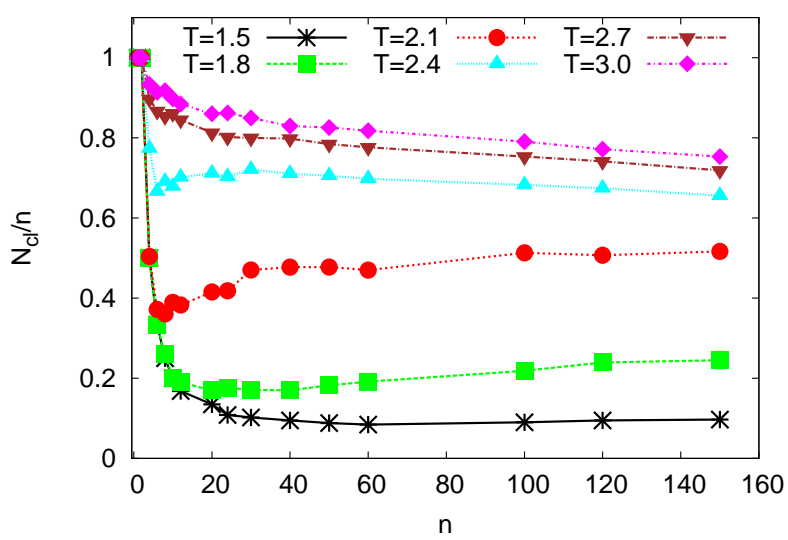

Fig. 6 Average number of clusters $N_{c l}$ divided by the total nunber of blocks ( $n$ ) versus $n$ for different temperatures, as indicated.

ber of monomers per block is small $(N=4$ to $N=1)$. When the temperature increases, we see that the plateau for small $n$ becomes shorter and for temperatures $T>2.4$ has completely disappeared. This shows that for $T>2.4$ the effect of the temperature on the behavior of the multiblock chain is minor and its behavior is defined by the incompatibility between species $\mathrm{A}$ and $\mathrm{B}$. We conclude that although the number of contacts A-B increases with increasing $n$, the effect of the temperature (when $T<2.4$ ) keeps the number of overall contacts almost constant for $N>30$. At temperature $T>2.1$ the curves show a monotonous decrease in the number of contacts and a slight increase for high $n$. At temperature $T=3.0$ the variation of $n$ in multiblock chains has a very small effect and the difference between $n=1$ and $n=2$ is rather indistinguishable.

A better understanding of all the above effects can be achieved in terms of the analysis of the formed clusters from blocks of the same type. In Fig. 6 we have computed the average number of clusters with blocks of type A together with the different clusters of type B. Of course, when $n=1$ we have only one cluster with one type of monomers, and for $n=2$ we have always one cluster with monomers of type $\mathrm{A}$ and one cluster with monomers of type B and each cluster comprises only one block. For small $n$ the ratio $N_{c l} / n$ should be one. At temperature $T=1.5, N_{c l} / n$ stays zero up to $n=12$. This plateau regime starts at smaller $n$ as the temperature increases, in accordance with the results of our previous figures. For $n>30$ the ratio $N_{c l} / n$ stays almost constant showing that the effect of the temperature takes over the effect of the incompatibility between the monomers of different type. At higher temperatures, i.e., $T=1.8$ and $T=2.1, N_{c l} / n$ slightly increases. For even higher temperatures a monotonic behavior is observed. The highest variations are observed for high $N$, where the ratio $N_{c l} / n$ decreases rather sharply.

We can go one step further in order to understand the above

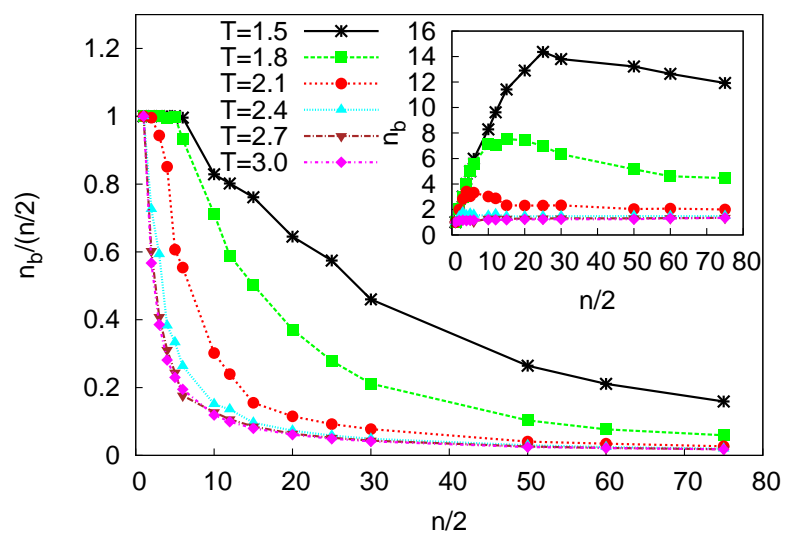

Fig. 7 Number of blocks $n_{b}$ in each cluster (A or B) divided with the number $n / 2$ of $\mathrm{A}$ (or $\mathrm{B}$ ) blocks versus this number $(n / 2)$. The inset shows the number of blocks in a cluster (A or B) versus $n / 2$.

phenomena. We analyze the number of blocks $n_{b}$ of type A (or B) in a cluster of type A (or B), respectively. Then, the number of blocks $\mathrm{A}$ is $n / 2$. Correspondingly the number of blocks with monomers of type $\mathrm{B}$ is $n / 2$. Due to the symmetry of our model we expect the same results for the analysis of clusters with blocks of type A and of clusters with blocks of type B. Indeed, our results show that this is true confirming the equilibration procedure, which was adopted in this study. In Fig.7 we show such plots where the number of blocks of a cluster is plotted versus the total number of A (or B) blocks of the multiblock chain. It is now clearly seen the range of $N$ where all blocks of the same type belong to the same single cluster. This happens when $n_{b} /(n / 2)=1$. When the temperature is rather low $(T=1.5)$ this plateau extends up to values $n=20$, which corresponds to block length of $N=30$. This means that the multiblock chain is separated in two clusters, that is one of type A and another of type B. This case then corresponds to the chain of $n=20$, and this explains the results of Figs. 5 and 6 When the temperature is lower, the number of blocks in the clusters is higher and therefore the curve corresponding to $T=1.5$ shows a smooth gradual decrease. More abrupt is the change in higher temperatures where the ratio $n_{b} /(n / 2)$ decreases abruptly. Then the plateau that follows with increasing $n$ shows the range of $n$ that the enthalpic interactions take over. For $T>2.4$ the chains exhibit almost the same behavior with a sharp decrease at small $n$ and a plateau at higher $n$. This shows that at high temperatures and high $n$ hardly ever two blocks of the same type come together to form a cluster. Then the ratio $n_{b} /(n / 2) \approx 1 /(n / 2)$. However, at temperature $T=1.5$ this ratio has the value $n_{b} /(n / 2) \approx 0.2$ showing that the formation of clusters with more than two blocks is possible. The inset shows the above results in a different representation. Then the linear curve $(y=a x)$ denotes the regime that 


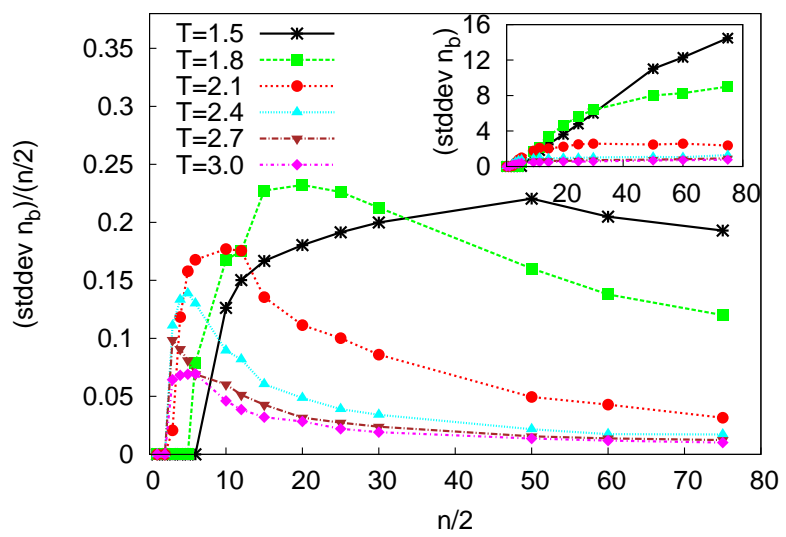

Fig. 8 Standard deviation in the number of blocks in each cluster (A or B) divided with the number $n / 2$ of A (or B) blocks that the multiblock chain contains versus this number $(n / 2)$. The inset shows the standard deviation in the number of blocks in a cluster (A or B) versus $n / 2$.

the ratio $n_{b} /(n / 2)=1$. It is also seen that this regime becomes smaller as the temperature is increased. Then, at low temperatures, the incompatibility between the monomers of different type becomes significant. This is not seen at high temperatures close to Theta, where the effect of the solvent quality and the enthalpic interactions rather cancel each other.

The number of blocks contained in a cluster is not the same, but it is a quantity that fluctuates, as we have already seen from Fig. 3 Then Fig. 8 shows these fluctuations of the number of blocks in a cluster. For small values of $n$ (or high $N$ ) such fluctuations should not exist, since there are only two clusters, each one with blocks of monomers of different type. For intermediate values of $n$ the fluctuations in the number of blocks in the clusters become rather high. The blocks of type A try to rearrange in such way that they form a single cluster, but the number $n$ is high enough to hinder such effect. For $n$ in the regime of strong fluctuations the formation of single clusters containing all blocks of the same type is possible. For higher $n$ the curves show a monotonic smooth behavior and the enthalpic interactions take over. It is also noticeable that the most pronounced fluctuations are observed in lower temperatures, where the effect of the incompatibility between different monomer types and the effect of the solvent incompatibility compete. At $T=1.5$ the fluctuations persist over high values of $n$. This is clearly seen from the inset of Fig. 8 Also, as $N$ becomes smaller, the fluctuation in the number of blocks in a cluster is higher.

Interesting is the dependence on the temperature for this particular case. From Fig. 9 we can clearly distinguish three different cases. For small values of $n(2<(n / 2)<6)$ the formation of a single cluster is possible up to temperatures

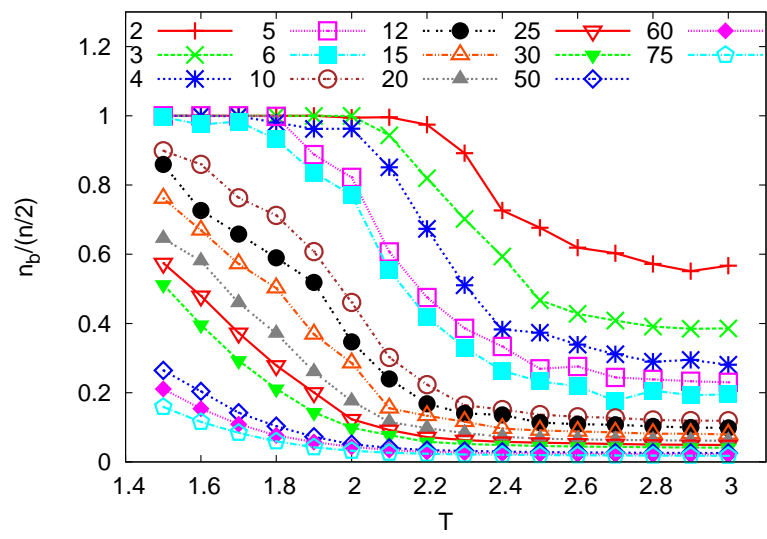

Fig. 9 Number of blocks $n_{b}$ in each cluster (A or B) divided with the number $n / 2$ of $\mathrm{A}$ (or B) blocks that the multiblock chain contains versus temperature.

$T=1.8$. In this case the blocks of the same type belong always to the same cluster. This behavior is weakened as $n$ increases, or correspondingly $N$ decreases. The second case is that for $10<(n / 2)<30$, where the formation of clusters containing all blocks of the same type is possible. Of course, this is more probable when $N$ is higher and the temperature is low. As the temperature is increased, we obtain a strong decrease in the number of blocks per cluster and at high temperatures $T>2.3$ we obtain a plateau where $n_{b} /(n / 2) \approx 2 / n$. When $n$ is high the dependence on the temperature is rather small and the behavior of the chain is defined by the incompatibility between $\mathrm{A}$ and $\mathrm{B}$ monomers. Of course for the latter case the formation of single clusters with all blocks of the same type is impossible.

Figure 10 shows the dependence of the fluctuation in the number of blocks per cluster. The stronger fluctuations are observed at lower temperatures (depending on $n$ ) in the range between $T=1.7$ and $T=2.4$. As $n$ increases this peak of the higher fluctuations is shifted to the left at lower temperatures. The results of Figs. 9 and 10 show that at low temperatures the formation of single clusters is possible, but the same time the fluctuations in the number of blocks in the cluster are rather high.

For block copolymers we know that the incompatibility between different monomers is expressed by the term $\chi N$. Here, we discuss the effect of the block length $N$. We choose three different chain lengths, which correspond to low $N$, intermediate, and high enough, where always the blocks of the same type form a single cluster. Then we look for the dependence on $n$. The results of Fig. 111 confirm the above argument that the phase separation depends strongly on $N$, as expected, and rather weakly on $n$ for a collapsed globule. Of course the influence of the surface energy $\left(\propto R^{2}\right.$, with $R$ being the radius 


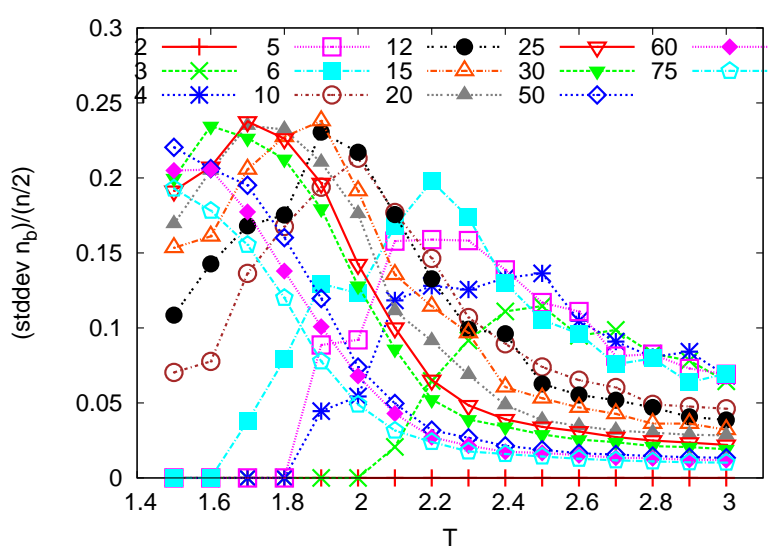

Fig. 10 Standard deviation in the number of blocks $n_{b}$ in each cluster (A or B) divided with the number $n / 2$ of $\mathrm{A}$ (or B) blocks that the multiblock chain contains versus temperature.

of the almost spherical collapsed object) is a combination of the above two parameters. $n$ has such effect with $N$ having the additional effect of controlling the incompatibility between $\mathrm{A}$ and B monomers. When $N$ is high $(N=80$ or 160$)$, the blocks are joined together into a single cluster with monomers of the same type at temperature $T=1.5$. Therefore, for this case no dependence on $n$ is observed. However, when $n$ becomes larger than some value, the formation of more than one cluster takes place. Then the overall length of the multiblock chain is very long and difficult to study with simulation techniques. Thus, such effect is not discussed in this manuscript and is not systematically studied. For intermediate $N(N=20)$ and small $n$ the formation of a single cluster is still possible, but this length is not high enough to ensure strong incompatibility between the blocks with monomers of different type. Somewhat richer behavior is expected as it has already been discussed, with strong fluctuations in the number of blocks per cluster. For $N=5$ the formation of a single cluster is not seen and the number of blocks per cluster and per block decreases reaching a plateau value at high $n$. For $T=3.0$ we see that that the different block length does not play any role. The three different block lengths have collapsed into a single curve $(\chi N \propto \Delta \varepsilon / T)$. This is the indication that the behavior of our multiblock chain depends strongly on the effect of the temperature. At high temperature also the curve reaches a plateau, that is for $(n / 2)>25$.

Figure 12 shows the dependence of the number of blocks per cluster on $N$. For $(n / 2)=4$ and at low temperature we have the formation of a single cluster for $N>20$. As the temperature increases this happens for higher values of $N$. At $T=2.2$ the curve takes gradually a constant value. For temperature $T=3.0$, which corresponds roughly to the Theta temperature, there is no dependence on $N$, as it is expected. This

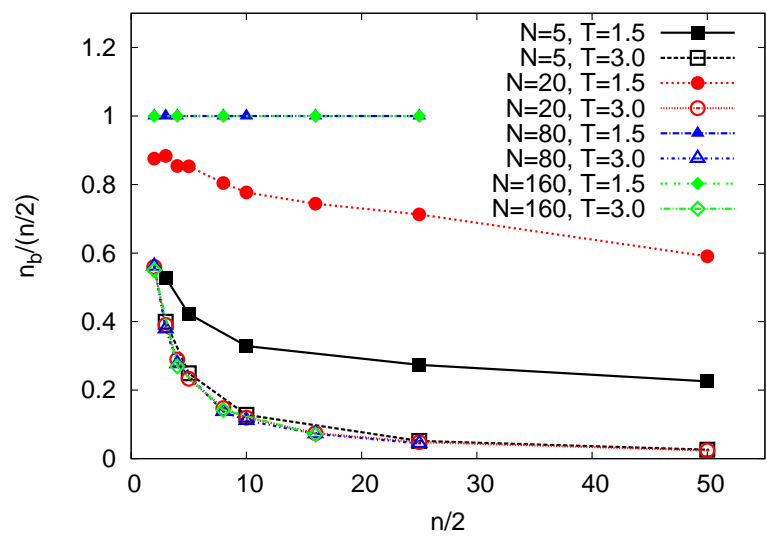

Fig. 11 Number of blocks $n_{b}$ per cluster divided with the number of blocks of type A (or B) versus this number of blocks $n / 2$. Results concern different block lengths $N$ and temperatures $T$.

is also observed for $n=20$ and $n=100$. Comparing the results for different $n$ at the low-temperature regime, we see that the phase separation depends rather strongly on $N$ and rather weakly on $n$. We should note here that all phase changes occur gradually due to the presence of thermal fluctuations in a collapsed object with a high ratio of surface to bulk energy.

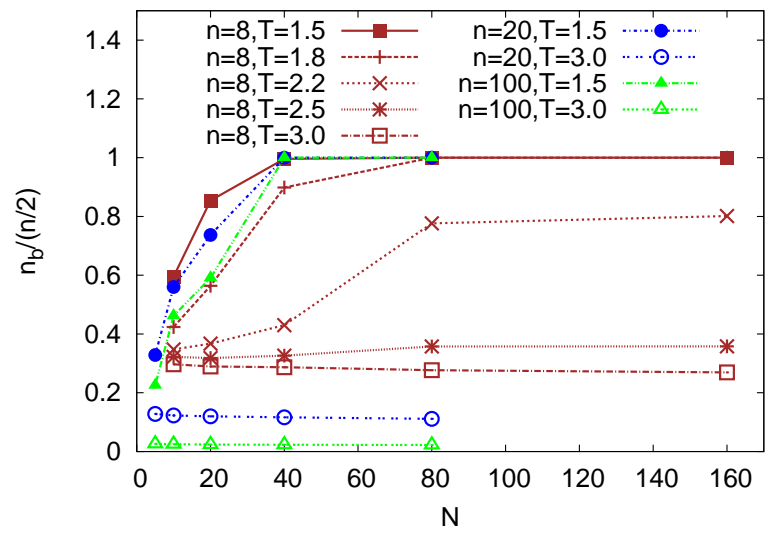

Fig. 12 Same as Fig.11, but the dependence on the block length $N$ is shown. The curves correspond to different numbers of blocks $n$ and temperatures $T$.

\section{Conclusions}

In this manuscript we have presented a detailed study of the phase behavior of multiblock copolymers varying the number of blocks $n$, the length of the blocks $N$, and the solvent quality by variation of the temperature. We have described the different types of phase separation appearing in linear multi- 
block copolymers and we have discussed in detail the effect of these parameters. Although the temperature (solvent incompatibility) and the block length $N$, as in the bulk, control the phase separation between blocks of different type (for given $\Delta \varepsilon)$, the effect of the number of blocks $n$ is also important due to the increase of the surface free energy. In the range of values that have been discussed here, very reach behavior is observed for intermediate values of $n, N$ and $T$, where the fluctuations in the number of blocks per cluster are pronounced. In this case the interplay between enthalpic and entropic effects compete resulting in different types of microphase separation in the collapsed states. The behavior of multiblock copolymers can be parallelized with that of various biological macromolecules which are formed by periodically repeated structural units ("monomers") along their chain. Our study provides a first step towards the understanding of the phase separation of such complex systems.

Acknowledgments One of us (P.E.T.) gratefully acknowledges financial support through a Max Planck fellowship awarded by the Max Planck Institute for Polymer Research.

\section{References}

1 H. Benoit, J. Polym. Sci. 11, 597 (1953)

2 P.J. Flory and A. Abe, Macromolecules 6, 1119 (1978)

3 O. Glatter and O. Kratky, Small Angle X-Ray Scattering (Academic Press, New York, 1982)

4 T.E. Schichtel and K. Binder, Macromolecules 20, 1671 (2987)

5 P.-G. de Gennes, Scaling Concepts in Polymer Physics (Cornell University Press, Ithaca, London, 1988)

6 L. Leibler, Macromolecules 13, 1602 (1980)

7 H. Benoit and G. Hadziioannou, Macromolecules 21, 1449 (1988)

8 A.M. Mayes and M. de la Cruz, J. Chem. Phys. 91, 7228 (1989)

9 M. Löwenhaupt and G.P. Hellmann, Polymer 32, 1065 (1991)

10 M. Matsen and M. Schick, Phys. Rev. Lett. 72, 2660 (1994)

11 M.W. Matsen and M. Schick, Macromolecules 27, 6761 (1994)

12 M.W. Matsen, J. Chem. Phys. 102, 3884 (1995)

13 G.H. Fredrickson and S.W. Sides, Macromolecules 36, 5415 (2003)

14 I. Erukhimovich and A.V. Dobrynin, Makromol. Symp. 36, 5415 (1994)

15 H. Angerman, G. ten Brinke and I. Erukhimovich, Macromolecules 29, 3255 (1996)

16 A.V. Dobrynin and L. Leibler, Macromolecules 30, 4756 (1997)

17 J.J.M. Slot, H.J. Angerman and G. ten Brinke, J. Chem. Phys. 108, 8677 (1998)

18 P. Friedel, A. John, R.R. Netz, D. Pospiech and D. Jehnichen, Macromol. Theory Simul. 11, 785 (2002)

19 A. John, P. Friedel, D. Pospiech, D. Jehnichen and C. Kunert, Macromol. Theory Simul. 13, 702 (2004)
20 H.J. Angerman, G. ten Brinke and J.J.M. Slot, Eur. Phys. J. B 12, 397 (1999)

21 L. Leibler and H. Benoit, Polymer 22, 195 (1981)

22 K.M. Hong and J. Noolandi, Polym. Commun. 25, 265 (1984)

23 G.H. Fredrickson, Macromolecules 24, 3456 (1991)

24 G.H. Fredrickson, S.T. Milner and L. Leibler, Macromolecules 25, 6341 (1992)

25 G. Hadziioannou, H. Benoit, W. Tang, K. Shull and C. Han, Polymer 33, 4677 (1992)

26 H. Angerman, G. Hadziioannou and G. ten Brinke, Phys. Rev. E 50, 3803 (1994)

27 R. Spontak and J. Zelinski, Macromolecules 25, 663 (1992)

28 R. Spontak, Macromolecules 27, 6363 (1994)

29 R. Spontak, J. Fung, M. Braunfeld, J. Sedat, D. Agrad, A. Ashaf and S. Smith, Macromolecules 29, 2850 (1996)

30 T. Hashimoto, T. Ogawa, N. Sakamoto, M. Ichimiya, J.K. Kim and C.D. Han, Polymer 39, 1573 (1998)

31 K. Sugiyama, O. Toshiyuki, A.A. El-Magd and A. Hirao, Macromolecules 43, 1403 (2010)

32 K. Matyjaszewski and J.H. Xia, Chem. Rev. 101, 2921 (2001)

33 L.A. Molina, A.L. Rodriguez and J.J. Freire, Macromolecules 27, 1160 (1994)

34 L.A. Molina and J.J. Freice, Macromolecules 28, 2705 (1995)

35 C.H. Vlahos, A. Horta, L.A. Molina and J.J. Freice, Macromolecules 27, 2726 (1994)

36 X.F. Yuan and A.J. Masters, Polymer 38, 339 (1997)

37 K.A. Dill, Biochemistry 24, 1501 (1985).

38 K.F. Lau and K.A. Dill, Macromolecules 22, 3986 (1989)

39 K.F. Lau and K.A. Dill, J. Chem. Phys. 95, 3775 (1991)

40 D. Shortle, H.S. Chan and K.A. Dill, Protein Sci. 1, 201 (1992)

41 K. Yue and K.A. Dill, Phys. Rev. E 48, 2267 (1993)

42 K. Yue and K.A. Dill, Proc. Natl. Acad. Sci. 92, 146 (1995)

43 J.E. Magee, V.R. Vasquez and L. Lue, Phys. Rev. Lett. 96, 207802 (2006)

44 O.F. Olaj, B. Neubauer and G. Zifferer, Macromol. Theory Simul. 7, 171 (1998)

45 G.S. Grest and K. Kremer, Phys. Rev. A 33, 3628 (1986)

46 G.S. Grest and M. Murat, Monte Carlo and Molecular Dynamics Simulations in Polymer Science, edited by K. Binder, Oxford University Press, New York, 1995

47 G.S. Grest, Adv. Polym. Sci. 138, 149 (1999)

48 S.K. Das, J. Horbach and K. Binder, J. Chem. Phys. 119, 1547 (2003)

49 G.S. Grest and M. Murat, Macromolecules 26, 3108 (1993)

50 http://www.gromacs.org

51 P.E. Theodorakis, W. Paul and K. Binder, Macromolecules 43, 5137 (2010)

52 P.E. Theodorakis, W. Paul and K. Binder, Europhys. Lett. 88, 63002 (2009)

53 P.E. Theodorakis, W. Paul and K. Binder, J. Chem. Phys. 133, 104901 (2010)

54 W.F. van Gunsteren and H.J.C. Berendsen, Mol. Simul. 1, 173 (1988)

55 F.H. Stillinger, J. Chem. Phys. 38, 1486 (1963) 